\title{
ON MONOTONE UNIONS OF MANIFOLDS
}

\author{
P. H. DOYLE ${ }^{1}$
}

If $U$ is a connected separable metric space that is locally euclidean of dimension $n, U$ is an $n$-manifold. We do distinguish $n$-manifolds and $n$-manifolds with boundary. By $\mathscr{T}(U)$ we denote the collection of all $n$-manifolds $M^{n}$ such that $M^{n}=\cup_{1}^{\infty} U_{i}$, where $U_{i} \subset U_{i+1}$ and each $U_{i}$ is a topological copy of $U$. The size of the collection is quite variable and we list some examples from which obvious questions arise.

1. If $S^{2}$ is the 2 -sphere and $E^{1}$ the real line, let $U=S^{2} \times E^{1}$. In this case $\mathfrak{N}(U)$ has two members, $U$ and $E^{3}$, where $E^{3}$ is euclidean 3 -space.

2. Let $M^{n}$ be a combinatorial compact $n$-manifold with $n \neq 4$. If $U$ is the complement of a flat $n$-cell in $M^{n}$, then $\Re(U)$ has $U$ as its only member [5]. The same result when $U$ is a cell follows from [2].

3 . If $U$ is $S^{2}$ with an infinite convergent sequence of points removed, then $\mathscr{T}(U)$ consists of exactly the proper open connected sets (proper domains) in $S^{2}$.

A manifold such as $U$ in example 2 above for which $\mathscr{T}(U)$ consists of one element is said to have the monotone union property. If $M^{n}$ is an $n$-manifold, then a standard decomposition of $M^{n}$ is a representation of $M^{n}$ as a disjoint union, $M^{n}=P \cup R$, where $P=E^{n}$ and $\operatorname{dim} R \leqq n-1$ [3].

THEOREM 1. Let $M^{n}$ be a compact $n$-manifold and $M^{n}=P \cup R a$ standard decomposition of $M^{n}$. If $U \supset R$ is any proper topological $n$ manifold in $M^{n}$, then $U$ has the monotone union property only if $U=M^{n}$ $-x$, where $x$ is a point in $M^{n}$.

Proof. Suppose $U \supset R$ is given and $U$ has the monotone union property. Let $x$ be a point in $M^{n}-U$. Then if we select a small open $n$-cell about $x, C^{n}$, with a flat boundary it follows that there is a homeomorphism of $M^{n}-x$ into $M^{n}-x$ that is fixed outside $\mathrm{Bd} C^{n}$ and the image fails to meet a neighborhood of $x$ in $M^{n}$. Thus we may assume that $U$ is not dense in $M^{n}$.

By the construction in [3] it follows that there is a bicollared $(n-1)$-sphere $S^{n-1}$ in $U, S^{n-1}$ is the boundary of a flat $n$-cell $K$ in $P$ that contains the point $x$ in its interior. If one now places a flat $n$-cell $K^{1}$ about $x$ in Int $K^{1}, K^{1} \subset M^{n}-U$, it can be seen that $M^{n}-x$ is a monotone union of copies of $U$. Thus if $U$ has this property $U=M^{n}-x$.

Received by the editors August 3, 1966.

1 The work was supported by the National Science Foundation Grant GP-7126. 
TheOREM 2. Let $M^{n}$ be a topological $n$-manifold and $C \neq \varnothing$ a compact proper subset of $M^{n}$. If $M^{n}-C$ is a manifold while $C$ lies in an open $n$-cell in $M^{n}$, then $M^{n}-C$ has the monotone union property if and only if $M^{n}$ less a point has this property and $C$ is cellular in $M^{n}$.

Proof. The sufficiency is trivial, let $M^{n}-C$ have the monotone union property. By hypothesis we may assume that $C$ lies in the interior of a flat $n$-cell $C^{n}$ in $M^{n}$.

Now if $C$ is not cellular in $M^{n}$, let $A$ be a tame arc in Int $C^{n}$ with endpoints $a$ and $b$, while $A \cap C=a$. At $b$ introduce a flat $n$-cell $K$ so that $K \cap A=b, K \cap C=\varnothing, K \subset$ Int $C^{n}$, and $K \cup A$ is a tame set. If $C^{1}=C \cup A \cup K$, we have $M^{n}-C=M^{n}-C^{1}$; there is a homeomorphism $h_{1}$ of $M^{n}$ onto $M^{n}$ such that $h_{1}\left(C^{n}\right)=K$ while the diameter of $h_{1}\left(C^{1}\right)$ $<\frac{1}{2}$. By repeating the construction it is clear that by induction we obtain $M^{n}$ less a point as a monotone union of copies of $M^{n}-C$. So $M^{n}-C=M^{n}-x$, where $x$ is a point and $C$ is cellular.

In [5] the sets considered are piecewise linear compact $n$-manifolds with $(n-1)$-sphere boundaries. For $n \neq 4$ it is shown that their interiors have the monotone union property. The cells among these spaces have the property that each carries a pair of disjoint copies of itself. We show that this is a peculiarity of cells among the spaces for $n \geqq 5$.

Lemma 1. Let $M^{n}$ be a compact n-manifold with an (n-1)-sphere boundary, $n \geqq 3$. If in Int $M^{n}$ there is a topological copy $T^{1}$ of $M^{n}$, then in Int $T^{1}$ there is a topological copy $T$ of $M^{n}$ and $X=M^{n} / T$ is a simply connected n-manifold with an (n-1)-sphere boundary.

Proof. In $T^{1}, \quad \mathrm{Bd} T^{1}$ is collared [1]. Thus we may select a sphere in this collar that bounds a copy $T$ of $M^{n}$ in Int $T^{1}$, $\operatorname{Bd} T$ is bicollared; then $X=M^{n} / T$ is an $n$-manifold with an $(n-1)$ sphere boundary. If $\pi_{1}(X) \neq 1$, we note that by the Van Kampen theorem

$$
\pi_{1}\left(M^{n}\right) \cong \pi_{1} \overline{\left(M^{n}-T\right)} * \pi_{1}(T),
$$

the free product. But

$$
\pi_{1}(X) \cong \pi_{1} \overline{\left(M^{n}-T\right)}
$$

Clearly $\pi_{1}\left(M^{n}\right)$ is then not finitely generated.

Corollary. If $\pi_{1}\left(M^{n}\right) \neq 1, M^{n}$ cannot contain two disjoint copies of itself. 
By these observations we note that if $M^{n}$ is as in Lemma 1 and contains two disjoint copies of itself, then $\pi_{1}\left(M^{n}\right)=1$.

We conclude that $M^{n}$ is homologically trivial by an argument on homology analogous to the above argument on $\pi_{1}\left(M^{n}\right)$.

THEOREM 3. Let $M^{n} \neq 4$ be a compact topological $n$-manifold with an $(n-1)$-sphere boundary. If $M^{n}$ contains two disjoint topological copies of itself, $M^{n}$ is a closed $n$-cell.

Proof. Since $M^{n}$ is contractible, if one sews an $n$-cell into $M^{n}$ by a homeomorphism on the boundary of the cell to that of $M^{n}$, the result is a $n$-sphere by [6] for $n \neq 3$ or 4 . Thus $M^{n}$ is an $n$-cell. In the case $n=3$, the Theorem follows from [4].

\section{REFERENCES}

1. M. Brown, "Locally flat embeddings of topological manifolds," pp. 83-91, in Topology of 3-manifolds and related topics, Prentice-Hall, Englewood Cliffs, N. J., 1962.

2. - The monotone union of open n-cells in an open n-cell, Proc. Amer. Math. Soc. 12 (1961), 812-814.

3. P. H. Doyle and J. G. Hocking, A decomposition theorem for $n$-dimensional manifolds, Proc. Amer. Math. Soc. 13 (1962), 469-471.

4. H. Kneser, Geschlossen Flachen in dreidimensional Mannig-faltigkeiten, Jber. Deutsch. Math.-Verein. 38 (1929), 248-260.

5. K. W. Kwun, Open manifolds with the monotone union property, Proc. Amer. Math. Soc. 17 (1966), 1091-1093.

6. M. H. A. Newman, The engulfing theorem for topological manifolds, Ann. of Math. 84 (1966), 553-571.

Michigan State University 